\title{
Preoperative ultrasound-guided multilevel paravertebral blocks reduce the incidence of postmastectomy chronic pain: a double-blind, placebo-controlled randomized trial
}

This article was published in the following Dove Medical Press journal: Journal of Pain Research

\author{
Bin Qian ${ }^{1, *}$ \\ Shiwei $\mathrm{Fu}^{2, *}$ \\ Yusheng Yao ${ }^{3,4}$ \\ Daoyi $\operatorname{Lin}^{3}$ \\ Li Huang ${ }^{3}$ \\ 'Department of Anesthesiology, \\ People's Hospital Affiliated to Fujian \\ University of Traditional Chinese \\ Medicine, Fuzhou, Fujian, China; \\ ${ }^{2}$ Department of Pathology, Union \\ Hospital of Fujian Medical University, \\ Fuzhou, Fujian, China; ${ }^{3}$ Department \\ of Anesthesiology, Shengli Clinical \\ Medical College of Fujian Medical \\ University, Fuzhou, Fujian, China; \\ ${ }^{4}$ Department of Anesthesiology, Fujian \\ Provincial Hospital, Fuzhou, Fujian, \\ China
}

*These authors contributed equally to this work
Correspondence: Yusheng Yao

Department of Anesthesiology, Shengli

Clinical Medical College of Fujian

Medical University and Department of Anesthesiology, Fujian Provincial Hospital, No. 134, Dongjie, Fuzhou 35000 I, Fujian, China

Tel +86 I35 59939629

Fax +86 59I 882I 7842

Email fjslyys@।26.com
Purpose: Chronic postsurgical pain is a challenging problem after breast cancer surgery. This prospective, randomized, double-blinded, parallel-group, placebo-controlled trial was conducted to evaluate the influence of preoperative ultrasound-guided multilevel paravertebral blocks (PVBs) on chronic pain following mastectomy.

Patients and methods: One hundred eighty-four women were randomized to receive ultrasound-guided multilevel (T1-T5) PVBs with $5 \mathrm{~mL}$ of ropivacaine $0.5 \%$ or normal saline per level. The primary end point was the incidence of chronic pain at 3 months following mastectomy assessed by the brief pain inventory (BPI), while the secondary end points were the acute postoperative pain, the number of patients requiring rescue analgesia, postoperative nausea and vomiting (PONV), side effects, and chronic pain at 6 months after surgery assessed by the BPI. Results: A total of 172 patients completed the study. Ultrasound-guided multilevel PVBs significantly decreased immediate postoperative pain for the first 12 hours $(P<0.001)$. Additionally, fewer patients in the PVB group required rescue analgesia in the first 48 hours postoperatively compared to the control group ( $5 / 86$ vs $28 / 86$, OR $=0.128,95 \%$ CI: $0.047-0.351$, $P<0.001)$. No statistically significant difference was tested between the two groups $(9.3 \%$ vs $17.4 \%$, OR $=0.419,95 \% \mathrm{CI}: 0.162-1.087, P=0.068$ ) in the incidence of PONV. At 3 months, the incidence of chronic pain (BPI average pain score $\geq 3$ ) was $34.5 \%$ and $51.2 \%$ (OR $=0.511$, 95\% CI: $0.277-0.944, P=0.031)$ in the PVB and control groups, respectively, and at 6 months, the incidence was $22.1 \%$ and $37.2 \%$ (OR $=0.479,95 \% \mathrm{CI}$ : $0.245-0.936, P=0.03)$, respectively. No complications occurred during the study.

Conclusion: This study indicated that perioperative ultrasound-guided multilevel PVBs with ropivacaine improved acute postoperative pain and decreased postmastectomy chronic pain at 3 and 6 months postoperatively.

Keywords: breast cancer surgery, multimodal analgesia, thoracic paravertebral block, acute postoperative pain, chronic postsurgical pain

\section{Introduction}

Breast cancer is the most common type of cancer and the leading cause of cancerrelated death among women. ${ }^{1}$ Acute postoperative pain is frequently moderate or even severe following breast cancer surgery. There is cumulative evidence that postoperative pain is closely associated with subsequent persistent pain lasting months. The International Association for the Study of Pain has defined chronic postsurgical pain (CPSP) as a pain that develops after surgical intervention and persists at least 2 months. ${ }^{2}$ 
The prevalence of CPSP has been reported in 25\%-60\% of patients after breast cancer surgery. ${ }^{3-5}$ Chronic postoperative pain has a large negative impact on the quality of life due to psychological distress and disability. ${ }^{6}$ Therefore, it has become an important clinical issue.

Thoracic paravertebral block (PVB) is one of the multimodal pain relief strategies, and the role of PVB in preventing chronic pain following breast cancer surgery has gained interest, but existing evidence is few and conflicting. ${ }^{7,8}$ In the literature, the technical aspect of PVB used varies considerably. The relatively high failure rates of landmark-guided PVB technique may contribute to methodological bias. ${ }^{9}$ The ultrasound-guided PVB technique was first described by Hara et al, ${ }^{10}$ which has been proved to improve regional and peripheral nerve block success rates. However, the effect of ultrasound-guided multilevel single-injection PVBs on CPSP after breast cancer surgery remains unclear.

We hypothesized that preoperative ultrasound-guided multilevel PVBs could provide effective analgesia during the perioperative period and may reduce postmastectomy chronic pain. Therefore, we designed this prospective randomized double-blind placebo-controlled trial to address the influence of ultrasound-guided multilevel PVBs on the incidence and intensity of CPSP after breast cancer surgery.

\section{Patients and methods Design and patients}

This prospective, randomized, double-blind, placebo-controlled trial was conducted in line with the Declaration of Helsinki and the CONSORT statement. The study protocol was approved by the Clinical Research Ethics Committee of Fujian Provincial Hospital (No. K2014-11-008) and registered at http://www.chictr.org.cn (ID: ChiCTRINR-16007874) on February 14, 2016. Written informed consent was obtained from each patient before recruitment. Female patients aged 18-60 years old with an American Society of Anesthesiologists physical status I or II, who were scheduled to undergo unilateral partial mastectomy with or without axillary lymph node dissection, were assessed for study eligibility. Exclusion criteria included the following: contraindications to PVB such as allergy to local anesthetics, infection, or coagulopathy; intake of analgesic within 24 hours before surgery; and unable to comply with the study protocol for any reason.

The randomization (1:1 ratio) was performed using a website random number generator (www.randomization. $\underline{\text { com). }}$. Assignments were concealed in sequentially numbered, opaque, sealed envelopes, and the envelopes were not opened until the day of the surgery. The consecutively enrolled patients were randomly assigned to either ropivacaine or saline PVB. All attending anesthesiologists, patients, and data collectors were unaware of treatment group assignment.

\section{Anesthetic procedure}

No premedication was given before anesthesia. Routine monitoring included electrocardiograph, pulse oximetry, capnography, noninvasive blood pressure, and temperature. Anesthesia was induced with sufentanil $0.5 \mu \mathrm{g} / \mathrm{kg}$ and propofol $2.0 \mathrm{mg} / \mathrm{kg}$. Laryngeal mask airway (LMA) intubation was facilitated with intravenous cisatracurium $0.15 \mathrm{mg} /$ $\mathrm{kg}$. After LMA intubation, the ventilation parameters were standardized (tidal volume $8-10 \mathrm{~mL} / \mathrm{kg}$, respiratory rate 12-15 bpm, a fraction of inspired oxygen 50\%) to achieve 35-45 mmHg of $\mathrm{P}_{\mathrm{ET}} \mathrm{CO}_{2}$. Anesthesia was maintained with an end-tidal sevoflurane $1.5 \%-3 \%$ to achieve the hemodynamic changes within $20 \%$ of baseline and a bispectral index of 40-60. Neuromuscular block was reversed by the administration of neostigmine $0.02 \mathrm{mg} / \mathrm{kg}$ and atropine 0.01 $\mathrm{mg} / \mathrm{kg}$, if necessary.

All patients were transferred to the postanesthesia care unit for 2 hours. Routine postoperative analgesia was administered including intravenous flurbiprofen axetil $50 \mathrm{mg}$ every 12 hours and sufentanil for patient-controlled intravenous analgesia (PCIA). The PCIA was programmed to deliver a bolus of sufentanil $2 \mu \mathrm{g}$ on demand with a 10 -minute lockout interval and a background infusion of sufentanil $2 \mu \mathrm{g} / \mathrm{h}$.

Postoperative pain at rest was rated using a numeric rating scale (NRS) of 0 (no pain) to 10 (worst pain imaginable). If the NRS score was $\geq 4$ or if the patient required, sufentanil $2 \mu \mathrm{g}$ was administered as a rescue analgesic by the PCIA device. Tropisetron $5 \mathrm{mg}$ plus dexamethasone $10 \mathrm{mg}$ was administered for nausea and vomiting prophylaxis after LMA insertion.

\section{Ultrasound-guided multilevel PVBs}

All patients received the ultrasound-guided PVBs between the first and fifth thoracic vertebrae in the prone position prior to surgery. This procedure was performed according to the methods described by the literature. ${ }^{11,12}$ The thoracic paravertebral space was scanned by ultrasonography (Model Edge; FUJIFILM SonoSite, Inc., Bothell, WA, USA) with a 2-5 MHz curved array ultrasound probe. The ultrasound probe was positioned in the vertical plane $\sim 2$ $\mathrm{cm}$ lateral to the spinous process. A $21 \mathrm{G}, 80 \mathrm{~mm}$, Uniplex Nanoline needle (PAJUNK GmbH Medizintechnologie, Geisingen, Germany) was advanced in an in-plane orientation 
toward the desired paravertebral space. Then, the needle was introduced to puncture the superior costotransverse ligament. After the position of the needle tip was confirmed, a total volume of $25 \mathrm{~mL}$ of ropivacaine $0.5 \%$ or normal saline ( 5 $\mathrm{mL}$ per level) was administered at the T1-T5 levels.

\section{Outcomes}

The primary end point was to address whether the use of ultrasound-guided multilevel PVBs with ropivacaine reduced the incidence of postmastectomy chronic pain 3 months after surgery. Chronic pain was defined by a score of $\geq 3$ on the single item (average pain) of the brief pain inventory (BPI). ${ }^{13,14}$ The BPI consists of three domains: 1) pain intensity (4 items), measured using an NRS of 0 (no pain) to 10 (worst pain imaginable); 2) pain's interference with daily activities (7 items), measured using a scale of 0 (no interference) to 10 (complete interference); and 3) percentage of relief provided by pain treatments, measured using a scale of 0 (complete relief) to 10 (no relief). A composite score of BPI ranging from 0 (optimal) to 120 (worst possible) was also recorded according to Initiative on Methods, Measurement, and Pain Assessment in Clinical Trials recommendations. ${ }^{15}$ A research nurse, who was blinded to treatment group, conducted a telephone interview with the patients at 3 and 6 months following mastectomy. Secondary end points were the acute postoperative pain, the number of patients requiring rescue analgesia, postoperative nausea and vomiting (PONV), side effects, and chronic pain at 6 months after surgery assessed by the BPI. Acute postoperative pain at rest was assessed using an NRS at $0.5,1$, $2,4,8,12,24$, and 48 hours following breast cancer surgery.

\section{Statistical analysis}

The sample size calculation was based on the primary end point (the incidence of chronic pain at 3 months). The estimated prevalence of chronic pain in the literature is $40 \%$ at 3 months. In order to detect a $50 \%$ reduction of the incidence of chronic pain (from $40 \%$ to $20 \%$ ), a sample size of 82 subjects per group would be required to achieve an alpha of 0.05 and obtain $90 \%$ power. Allowing for $10 \%$ of patients' incomplete follow-up or dropout, a population of 184 patients was required in this study.

All continuous data were tested for normality of distribution via the Shapiro-Wilk test. Parametric variables were reported as mean (SD) and analyzed with the two-sample independent $t$-test, and nonparametric variables were reported as median (IQR) and analyzed with the Mann-Whitney $U$-test. For the evaluation of postoperative NRS scores, we used a repeated measures analysis with post hoc testing.
Categorical variables were reported as number (percentage) and compared by the chi-squared or Fisher's exact test when necessary. All reported $P$-values are two-tailed, and a $P$-value of $<0.05$ was considered statistically significant.

\section{Results}

We investigated 207 patients for eligibility to participate in this study from March 12, 2016 to February 16, 2018 at Fujian Provincial Hospital. A CONSORT flow diagram describing the progression of subjects through the study is presented in Figure 1. Of the 207 patients, 9 did not meet the inclusion criteria and 14 declined to participate, and the remaining 184 patients were enrolled in this study. Two patients in the PVB group and one patient in the control group were later excluded due to protocol breach. In addition, four patients in the PVB group and five patients in the control group could not be reached for follow-up. Finally, 172 patients completed the study and their data were analyzed. Demographic characteristics and type and duration of surgery were not statistically different between groups (Table 1).

The acute postoperative pain scores during the first 48 hours following surgery at rest are shown in Figure 2. Briefly, the NRS scores were lower in the first 12 hours postoperatively in the PVB group compared to the control group $(P<0.001)$. Additionally, at 24 and 48 hours postoperatively, there was no difference detected between the groups in the NRS scores. As shown in Table 2, ultrasound-guided multilevel PVBs with ropivacaine decreased the number of patients requiring rescue analgesia for the first 48 hours $(5 / 86$ vs $28 / 86$, OR $=0.128,95 \%$ CI: $0.047-0.351, P<0.001)$. In addition, the incidence of PONV was $9.3 \%$ in the PVB and $17.4 \%$ in the control group, and no statistically significant difference was observed (OR $=0.419,95 \%$ CI: $0.162-1.087$, $P=0.068$ ). No episodes of PVB-related complications (such as local anesthetic toxicity, pneumothorax, epidural or subarachnoid injection) were observed.

The BPI average pain scores evaluated 3 and 6 months after surgery are presented in Figure 3. Correspondingly, the total of BPI scores at 3 and 6 months postoperatively in the PVB group was lower compared with the control group (both $P<0.001$, Table 2). At 3 months, the incidence of chronic pain (BPI average pain score $\geq 3$ ) was $34.5 \%$ and $51.2 \%$ in the PVB and control groups, respectively (Table 2), and patients in the PVB group had lower odds of developing chronic pain compared to the control group $(\mathrm{OR}=0.511,95 \% \mathrm{CI}$ : $0.277-0.944, P=0.031)$. Similarly, at 6 months, the incidence of chronic pain was $22.1 \%$ and $37.2 \%$ in the PVB and control groups, respectively (Table 2), and patients in the PVB group 


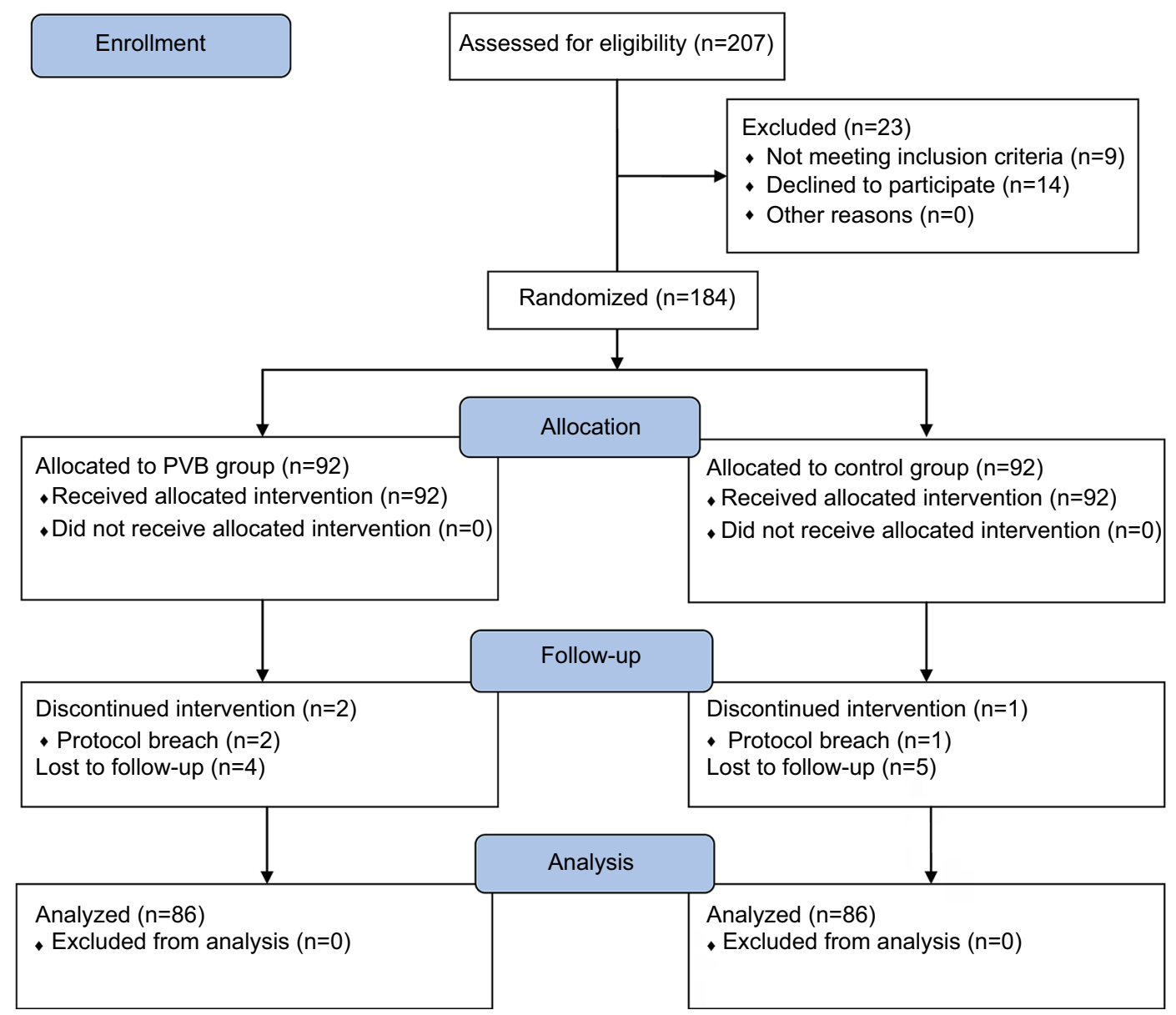

Figure I CONSORT flow diagram describing the progression of subjects through the study.

Abbreviation: PVB, paravertebral block.

Table I Demographic data and clinical characteristics of the patients in the study

\begin{tabular}{|l|l|l|l|}
\hline Variables & PVB group $(\mathbf{n = 8 6})$ & Control group $(\mathbf{n = 8 6})$ & $\boldsymbol{P}$-value \\
\hline Age (years) & $46.5 \pm 6.7$ & $44.8 \pm 7.2$ & 0.498 \\
Body weight $(\mathrm{kg})$ & $58.5 \pm 7.4$ & $60.2 \pm 7.8$ & 0.379 \\
BMI $\left(\mathrm{kg} / \mathrm{m}^{2}\right)$ & $23.9 \pm 4.4$ & $24.3 \pm 5.1$ & 0.713 \\
ASA physical status (I/II) & $31 / 55$ & $37 / 49$ & 0.349 \\
Duration of surgery (minutes) & $116 \pm 16$ & $128 \pm 17$ & 0.537 \\
Type of surgery & & & \\
$\quad$ Partial mastectomy with ALND & $34(39.5 \%)$ & $39(45.3 \%)$ & 0.44 \\
Partial mastectomy without ALND & $52(60.5 \%)$ & $47(54.7 \%)$ & \\
\hline
\end{tabular}

Note: Values are reported as mean \pm SD or number (percentage).

Abbreviations: ALND, axillary lymph node dissection; ASA, American Society of Anesthesiologists; BMI, body mass index; PVB, paravertebral block.

had lower odds of developing chronic pain than the control group $(\mathrm{OR}=0.479,95 \% \mathrm{CI}: 0.245-0.936, P=0.03)$.

\section{Discussion}

This prospective, randomized, double-blind, parallel-arm, placebo-controlled follow-up study suggested that preoperative ultrasound-guided single-injection multilevel PVBs significantly reduce acute postoperative pain and chronic pain after breast cancer surgery. Our results of the NRS of acute postoperative pain and the number of patients requiring rescue analgesia indicated that perioperative ultrasoundguided multilevel PVB can be used as a potent adjuvant for the multimodal management of pain following mastectomy. Although the effective duration of single-injection PVBs is 
$<24$ hours, the effect of PVBs against chronic pain can last for 3 and 6 months following mastectomy. Our findings have broad clinical implications considering that tens of thousands of women undergoing breast cancer surgery have a relatively high risk of developing chronic pain.

In this study, the score of intensity of acute postoperative pain at rest was mostly $<3$ on the NRS, and a statistically significant difference between the two groups was observed at least in the first 12 hours. These findings corroborate the existing literature and demonstrate PVBs improve postoperative pain control in patients undergoing mastectomy. ${ }^{16,17}$ In addition to alleviating acute postoperative pain, PVBs decrease the risk for the development of chronic pain. The BPI average pain scores and the total of BPI scores were evaluated at both 3- and 6-month follow-up as outcomes in this study. In general, 3 months is the earliest period for pain to be classified as chronic, ${ }^{18}$ but 6 months is more likely to allow enough time for recovery from swelling and acute postsurgical inflammatory processes. ${ }^{19}$ In this study, the overall incidence of chronic pain was $43 \%$ and $29.7 \%$ at 3 and 6 months, respectively, which is in line with the previous reports $(25 \%-60 \%))^{3-5}$ This wide range of prevalence of chronic pain in the literature is probably due to differences in the definitions of chronic pain considered, the methods used to evaluate pain intensity, time taken for CPSP assessment, and the type of surgery performed. The pathogenesis of CPSP is still not completely clear, but evidence continues to accumulate concerning the role of sensitization in chronic pain after surgery. ${ }^{20}$ The methods suggested for preventing CPSP include reduction of reversible peripheral and central neuronal sensitization, reduction of inflammatory responses, as well as use of minimally invasive operative procedures. ${ }^{21}$ Therefore, regional or peripheral nerve blockade is widely used to diagnose and treat chronic postoperative pain. Historically, in most cases, chronic pain after breast surgery has been attributed to nerve damage, which results in spinal

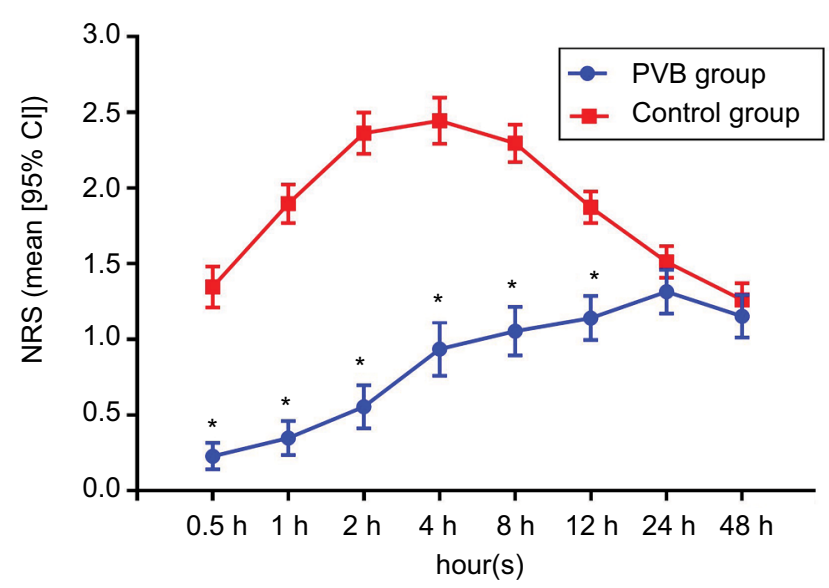

Figure 2 The NRS scores at rest during the first 48 postoperative hours. The NRS showed lower scores at rest in the first 12 hours after surgery in the PVB group than the control group $(* P<0.001)$.

Abbreviations: NRS, numeric rating scale; PVB, paravertebral block.

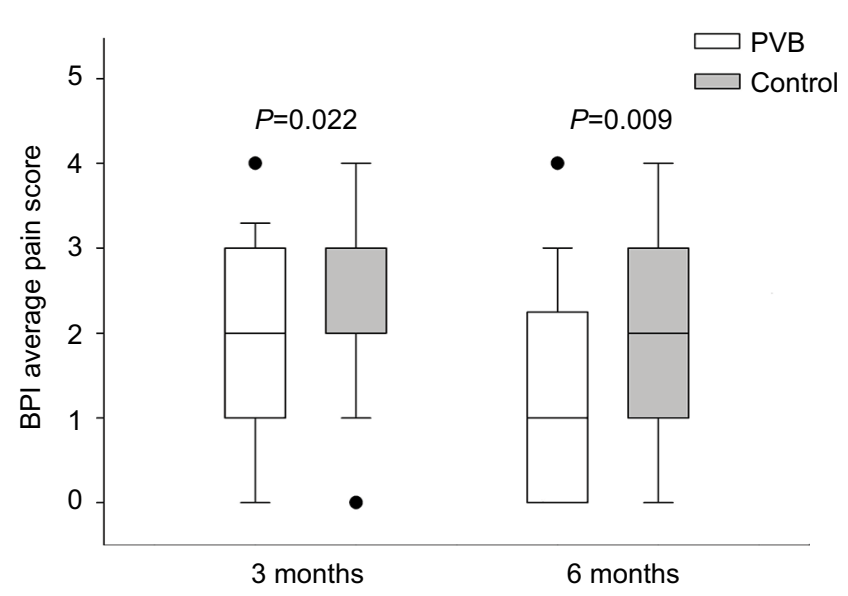

Figure 3 Box plots of BPI average pain score in patients receiving PVBs either with ropivacaine $0.5 \%$ or with normal saline. The median BPI pain score in the PVB group was lower compared with the control group at 3 and 6 months postoperatively ( $P=0.022$ and $P=0.009$, respectively).

Abbreviations: BPI, brief pain inventory; PVB, paravertebral block.

Table 2 Outcome measurements during the period of the study

\begin{tabular}{|l|l|l|l|}
\hline Variables & PVB group (n=86) & Control group (n=86) & P-value \\
\hline Incidence of chronic pain & & & \\
At 3 months & $30(34.9 \%)$ & $44(51.2 \%)$ & 0.03 I \\
At 6 months & $19(22.1 \%)$ & $32(37.2 \%)$ & 0.03 \\
BPI total score (0-120) & $7(14)$ & $18(17)$ & $<0.001$ \\
At 3 months & $5(9)$ & $8(19)$ & $<0.001$ \\
At 6 months & $5(5.8 \%)$ & $28(32.6 \%)$ & $<0.001$ \\
Number of patients requiring analgesics & $7(9.3 \%)$ & $15(17.4 \%)$ & 0.068 \\
PONV & & & \\
\hline
\end{tabular}

Note: Values are reported as median (IQR) or number (percentage).

Abbreviations: BPI, brief pain inventory; PONV, postoperative nausea and vomiting; PVB, paravertebral block. 
sensitization, expansion of sensory receptive fields, and alterations in the processing of innocuous stimuli. In this study, hyperalgesia did not develop when local anesthetic injection was administered before surgery. This mechanism may have contributed to less postoperative NRS pain scores in the PVB group, in which multilevel PVBs were inserted before incision.

PVBs have minimal effects on cardiovascular variables. No episodes of clinically significant hypotension or bradycardia were identified in this study, which may be attributed to the less sympathetic blockade and unilateral segmental nature of the block. In addition, although this study was not powered to detect the difference in the incidence of PONV, patients who received PVBs showed a beneficial trend, which may partly reflect the PVBs' opioid-sparing effect. Moreover, no block-related complications (such as local anesthetic toxicity, pneumothorax, epidural or subarachnoid injection) occurred during the study. Actually, the incidence of complications was very low, and ultrasound further provided significant improvements in regional anesthesia.

Several limitations of this study need to be addressed when interpreting the results. First, our study did not examine possible psychological factors influencing chronic pain. However, a previous report suggested that preoperative emotional variables (such as anxiety or depression) did not independently contribute to the risk of developing CPSP after surgery. ${ }^{22}$ Second, we conducted this study in a single hospital and patients were treated based on consistent guidelines. Thus, this may potentially limit the external validity of our findings. Third, our Research Ethics Committee suggested us that acute postoperative pain cannot be considered as a primary outcome and evaluating the pain intensity at movement may be unfavorable for the patients. Therefore, we only examined the acute postoperative pain at rest which caused a potential bias in this study. In addition, we did not confirm whether the PVBs were administered successfully by testing sensory blockade due to the blinded nature of the trial. However, administration of all the PVBs was performed using real-time ultrasound guidance by a single experienced anesthesiologist. Thus, we believe that the majority of the PVBs were successfully administered.

\section{Conclusion}

In summary, our findings suggest that perioperative ultrasound-guided single-injection multilevel PVBs with ropivacaine may be protective against CPSP at 3 and 6 months following breast cancer surgery.

\section{Data sharing statement}

The individual participant data underlying published results can be accessed with approval from the principal investigator after 6 months of publication of main results. The study protocol, statistical analysis plan, and clinical study report will also be available.

\section{Acknowledgments}

This study was supported by Fujian Medical Innovation Project (2015-CXB-24), Fujian Young and Middle-Aged Backbone Talents Training Project (2016-ZQN-7), and Fujian Joint Funds for the Innovation of Science and Technology (2017Y9066). The authors also thank Professor Yanqing Chen from the Department of Anesthesiology, Fujian Provincial Hospital, for her kind support and cooperation.

\section{Author contributions}

Yusheng Yao designed the study. Daoyi Lin and Li Huang collected the data. Shiwei Fu and Daoyi Lin performed the data analysis and statistical analysis. Yusheng Yao conducted the data interpretation. Bin Qian and Shiwei Fu prepared the manuscript. Yusheng Yao and Bin Qian performed the surgeries. All authors contributed to data analysis, drafting and revising the article, gave final approval of the version to be published, and agree to be accountable for all aspects of the work.

\section{Disclosure}

The authors report no conflicts of interest in this work.

\section{References}

1. Bray F, Ferlay J, Soerjomataram I, Siegel RL, Torre LA, Jemal A. Global cancer statistics 2018: GLOBOCAN estimates of incidence and mortality worldwide for 36 cancers in 185 countries. CA Cancer $J$ Clin. 2018;68(6):394-424.

2. VanDenKerkhof EG, Peters ML, Bruce J. Chronic pain after surgery: time for standardization? A framework to establish core risk factor and outcome domains for epidemiological studies. Clin J Pain. 2013;29(1):2-8.

3. Gärtner R, Jensen MB, Nielsen J, Ewertz M, Kroman N, Kehlet H. Prevalence of and factors associated with persistent pain following breast cancer surgery. JAMA. 2009;302(18):1985-1992.

4. Mejdahl MK, Andersen KG, Gärtner R, Kroman N, Kehlet H. Persistent pain and sensory disturbances after treatment for breast cancer: six year nationwide follow-up study. BMJ. 2013;346:f1865.

5. Bruce J, Thornton AJ, Powell R, et al. Psychological, surgical, and sociodemographic predictors of pain outcomes after breast cancer surgery: a population-based cohort study. Pain. 2014;155(2):232-243.

6. Meijuan Y, Zhiyou P, Yuwen T, Ying F, Xinzhong C. A retrospective study of postmastectomy pain syndrome: incidence, characteristics, risk factors, and influence on quality of life. Scientific World Journal. 2013;2013(2): $1-6$.

7. Hussain N, Shastri U, McCartney CJL, et al. Should thoracic paravertebral blocks be used to prevent chronic postsurgical pain after breast cancer surgery? A systematic analysis of evidence in light of IMMPACT recommendations. Pain. 2018;159(10):1955-1971. 
8. Heesen M, Klimek M, Rossaint R, Imberger G, Straube S. Paravertebral block and persistent postoperative pain after breast surgery: meta-analysis and trial sequential analysis. Anaesthesia. 2016;71(12):1471-1481.

9. Najarian MM, Johnson JM, Landercasper J, Havlik P, Lambert PJ, McCarthy D. Paravertebral block: an alternative to general anesthesia in breast cancer surgery. Am Surg. 2003;69(3):213-218.

10. Hara K, Sakura S, Nomura T, Saito Y. Ultrasound guided thoracic paravertebral block in breast surgery. Anaesthesia. 2009;64(2): 223-225.

11. O Riain SC, Donnell BO, Cuffe T, Harmon DC, Fraher JP, Shorten G. Thoracic paravertebral block using real-time ultrasound guidance. Anesth Analg. 2010;110(1):248-251.

12. Abdallah FW, Morgan PJ, Cil T, et al. Ultrasound-guided multilevel paravertebral blocks and total intravenous anesthesia improve the quality of recovery after ambulatory breast tumor resection. Anesthesiology. 2014;120(3):703-713.

13. Dworkin RH, Turk DC, Peirce-Sandner S, et al. Research design considerations for confirmatory chronic pain clinical trials: IMMPACT recommendations. Pain. 2010;149(2):177-193.

14. Albi-Feldzer A, Mouret-Fourme E E, Hamouda S, et al. A double-blind randomized trial of wound and intercostal space infiltration with ropivacaine during breast cancer surgery: effects on chronic postoperative pain. Anesthesiology. 2013;118(2):318-326.
15. Dworkin RH, Turk DC, Farrar JT, et al. Core outcome measures for chronic pain clinical trials: IMMPACT recommendations. Pain. 2005;113(1-2):9-19.

16. Parikh RP, Sharma K, Guffey R, Myckatyn TM. Preoperative paravertebral block improves postoperative pain control and reduces hospital length of stay in patients undergoing autologous breast reconstruction after mastectomy for breast cancer. Ann Surg Oncol. 2016;23(13):4262-4269.

17. Alfaro-de La Torre P, Fajardo-Pérez M. Thoracic paravertebral block and its effects on chronic pain and health-related quality of life after modified radical mastectomy. Reg Anesth Pain Med. 2015;40(2):177-178.

18. Classification of chronic pain. Descriptions of chronic pain syndromes and definitions of pain terms. Prepared by the International Association for the study of pain, Subcommittee on Taxonomy. Pain Suppl. 1986;3:S1-226.

19. Ugur S, Arıc1 C, Yaprak M, et al. Risk factors of breast cancer-related lymphedema. Lymphat Res Biol. 2013;11(2):72-75.

20. Gilron I, Kehlet H. Prevention of chronic pain after surgery: new insights for future research and patient care. Can JAnaesth. 2014;61(2):101-111.

21. Kehlet H, Jensen TS, Woolf CJ. Persistent postsurgical pain: risk factors and prevention. Lancet. 2006;367(9522):1618-1625.

22. Poleshuck EL, Katz J, Andrus $\mathrm{CH}$, et al. Risk factors for chronic pain following breast cancer surgery: a prospective study. $J$ Pain 2006;7(9):626-634.
Journal of Pain Research

\section{Publish your work in this journal}

The Journal of Pain Research is an international, peer reviewed, open access, online journal that welcomes laboratory and clinical findings in the fields of pain research and the prevention and management of pain. Original research, reviews, symposium reports, hypothesis formation and commentaries are all considered for publication

\section{Dovepress}

The manuscript management system is completely online and includes a very quick and fair peer-review system, which is all easy to use. Visit http://www.dovepress.com/testimonials.php to read real quotes from published authors. 